\title{
Scenario development for D-T operation at JET
}

L. Garzotti ${ }^{1}$, C. Challis ${ }^{1}$, R. Dumont ${ }^{2}$, D. Frigione ${ }^{3}$, J. Graves ${ }^{4}$, E Lerche $^{5}$, J. Mailloux ${ }^{1}$, M. Mantsinen ${ }^{6,7}$, F. Rimini $^{1}$, F. Casson $^{1}$, A. Czarnecka ${ }^{8}$, J. Eriksson ${ }^{9}$, R. Felton ${ }^{1}$, L. Frassinetti $^{10}$, D. Gallart ${ }^{6}$, J. Garcia ${ }^{2}$, C. Giroud ${ }^{1}$, E. Joffrin ${ }^{2}$, H.-T. Kim ${ }^{11}$, N. Krawczyk ${ }^{8}$, M. Lennholm ${ }^{11}$, P. Lomas ${ }^{1}$, C. Lowry $^{11}$, L. Meneses ${ }^{12}$, I. Nunes ${ }^{12}$, C. M. Roach ${ }^{1}$, M. Romanelli $^{1}$, S. Sharapov ${ }^{1}$, S. Silburn ${ }^{1}$, A. Sips ${ }^{11}$, E. Stefániková $^{9}$, M. Tsalas ${ }^{13}$, D. Valcarcel ${ }^{1}$, M. Valovič $^{1}$ and JET contributors*

${ }^{1}$ CCFE, Culham Science Centre, Abingdon, Oxon, OX14 3DB, UK

${ }^{2}$ CEA, IRFM, F-13108 Saint Paul Lez Durance, France

${ }^{3}$ Unit Tecnica Fusione - ENEA C. R. Frascati - via E. Fermi 45, 00044 Frascati (Roma), Italy

${ }^{4}$ Ecole Polytechnique Fédérale de Lausanne (EPFL), Swiss Plasma Center (SPC), CH-1015 Lausanne, Switzerland

${ }^{5}$ Laboratory for Plasma Physics Koninklijke Militaire School - École Royale Militaire Renaissancelaan 30 Avenue de la Renaissance B-1000, Brussels, Belgium

${ }^{6}$ Barcelona Supercomputing Center, Barcelona, Spain

${ }^{7}$ ICREA, Barcelona, Spain

${ }^{8}$ Institute of Plasma Physics and Laser Microfusion, Hery 23, 01-497 Warsaw, Poland ${ }^{9}$ Department of Physics and Astronomy, Uppsala University, SE-75120 Uppsala, Sweden

${ }^{10}$ Fusion Plasma Physics, EES, KTH, SE-10044 Stockholm, Sweden

${ }^{11}$ EUROfusion Programme Management Unit, Culham Science Centre, Culham, OX14 3DB, UK

${ }^{12}$ Instituto de Plasmas e Fusão Nuclear, Instituto Superior Técnico, Universidade de Lisboa, Lisboa, Portugal

${ }^{13}$ FOM Institute DIFFER, Eindhoven, the Netherlands

*See the author list of "E. Joffrin et al. 2019 Nucl. Fusion to be published"

Abstract. The JET exploitation plan foresees D-T operations in 2020 (DTE2). With respect to the first D-T campaign in 1997 (DTE1), when JET was equipped with a carbon wall, the experiments will be conducted in presence of a berylliumtungsten ITER-like wall (ILW) and will benefit from an extended and improved set of diagnostics and higher additional heating power (32 MW NBI + $8 \mathrm{MW} \mathrm{ICRH).}$ Among the challenges presented by operations with the new wall, there are a general deterioration of the pedestal confinement, the risk of heavy impurity accumulation in the core, which, if not controlled, can cause the radiative collapse of the discharge, and the requirement to protect the divertor from excessive heat loads, which may damage it permanently. Therefore, an intense activity of scenario development has been undertaken at JET during the last three years to overcome these difficulties and prepare the plasmas needed to demonstrate stationary high fusion performance and 
clear alpha particle effects. The paper describes the status and main achievements of this scenario development activity, both from an operational and plasma physics point of view.

PACS numbers: 52.55.Fa, 89.30.Jj, 52.55.-s 


\section{Introduction}

The JET exploitation plan foresees D-T operations in 2020 (DTE2) [1]. While in the first D-T campaign in 1997 (DTE1) one of the objectives was to maximize the neutron yield, even if transiently, a key element of this experimental campaign will be on demonstrating that such high neutron yield can be obtained in stationary conditions lasting for many confinement times. Demonstrating the readiness of JET to achieve this objective is a major activity of the campaigns in pure $\mathrm{D}$ and pure $\mathrm{T}$, which will be conducted before DTE2. In particular, JET performances will be measured against a series of key performance indicators, the most relevant of which, in terms of readiness for high-performance D-T operation, is the establishment of a reliable scenario capable of producing $5 \cdot 10^{16}$ neutrons/s for $5 \mathrm{~s}$ in D plasmas, averaged over the best 20 pulses. This would provide a solid basis for the target of $15 \mathrm{MW}$ of fusion power over $5 \mathrm{~s}$ in $\mathrm{D}-\mathrm{T}$. The progress towards this target is illustrated in figure 1, showing the average D-D neutron rate as a function of the averaging time.

It can be seen that, while JET has recovered the performance of the $\mathrm{C}$ wall for averaging times $>5 \mathrm{~s}$, it has not yet been possible to replicate the peak neutron rates achieved in the past over shorter time windows. It can also be seen that the milestone for the 2016 campaign was not met. However, the neutron rate achieved is consistent with the available NBI power being limited by operational restrictions on the maximum voltage allowed on the injector acceleration grid. These restrictions will be lifted before the next experimental campaign. Fast progress was made as soon as high power $(\mathrm{NBI}+\mathrm{ICRH}=25-33 \mathrm{MW})$ became reliably available. To achieve the key performance indicator for a $\mathrm{D}$ - $\mathrm{T}$ ready plasma mentioned above the neutron rate needs to be increased by a factor of about two. This will be attempted in the upcoming campaigns, when $\sim 40 \mathrm{MW}$ of additional heating power are expected to be consistently available. An equally important scientific objective of DTE2 is to conduct experiments aimed at documenting and demonstrating unequivocally the effect of alpha particle physics such as alpha particle heating and alpha particle destabilisation of toroidal Alfvèn eigenmodes (TAE). This requires the development of a dedicated plasma scenario to maximise TAE drive by alpha particles while minimising mode damping.

The fusion production targets of DTE2 represent an extension of the achievements of DTE1 $[2,3]$ where $16 \mathrm{MW}$ of fusion power were achieved transiently and $4 \mathrm{MW}$ in steady state. The possibility of extending the performance relies mainly on the increased additional auxiliary heating power available with respect to DTE1. In fact, in DTE2 32 MW of NBI and 6-8 MW of ICRH will be available, which represents approximately a factor of two increase in additional power with respect to the highest fusion performance plasmas in DTE1. Moreover, a wider and more powerful set of diagnostics will be available and will allow a more in-depth analysis of the experimental

results $[4,5]$. Among the new diagnostics there are, for example, the new high-resolution Thomson scattering [6], to measure the electron density and temperature profiles with higher spatial and temporal resolutions than were achieved using LIDAR in 1997 and 
a series of neutron diagnostics, including a time-of-flight neutron spectrometer [7], to diagnose in detail the spectrum of the D-T neutrons.

Another major difference between DTE1 and DTE2 is the installation on JET of a new ITER-like wall made of Be (limiters and main wall) and W (divertor), which has replaced the $\mathrm{C}$ wall in use at the time of previous D-T experiments. The new wall imposes constraints on plasma operations. In particular, the continuous heat load on the divertor tiles will have to be mitigated in order not to exceed the surface temperature limits, which could cause the melting of the tiles. The transient heat loads associated with ELMs do not constitute a concern for JET scenarios and can be neglected in the optimization process. However, the potential source of sputtered $\mathrm{W}$ from the divertor will have to be minimized and the accumulation of $\mathrm{W}$ in the plasma core will have to be avoided. Finally, the disruptivity of the scenario will have to be low enough to guarantee safe operation (below 20\%), disruptions will have to be mitigated using massive gas injection (MGI) and performance degrading MHD instabilities will have to be avoided, as well as those leading to disruptions.

To overcome the challenges highlighted above in an integrated fashion an intense activity of scenario development has been undertaken at JET during the last three years in preparation for the DTE2 campaign. So far, the preparation work has been conducted in pure $\mathrm{D}$, but a $\mathrm{T}$ campaign is planned before DTE2 to assess how the isotopic effects will affect the scenarios developed in D. Further insight useful for the scenario development will be provided by a small number of dedicated experiments in $\mathrm{H}$ and isotope mixtures (H-T and $\mathrm{H}-\mathrm{D})$.

The importance of isotope effects was already recognized already in DTE1, for example in the optimised shear scenario, the progress of which in D-T was hampered by the lower L-H threshold compared to pure D [8]. Moreover, the recent $\mathrm{H}$ campaign at JET also showed clear isotope effects, affecting in particular the H-mode pedestal, which plays a crucial role in determining the performnce of the core plasma $[9,10]$.

In this paper we describe the main results achieved so far both from the operational and the plasma physics point of view. In section 2 we describe the main results of the scenario development activity aimed at achieving steady-state, high-performance plasmas, in section 3 we present the main results of the experimental work conducted to develop a scenario suitable for the study of alpha particle physics and in section 4 we will draw some conclusions and illustrate the future plans leading to DTE2.

\section{Scenarios for stationary high fusion performance}

Two complementary lines of research are pursued to address the problem of developing a scenario suitable for sustained high D-T fusion power: the baseline scenario $\left(\beta_{N} \sim 1.8\right.$, $\left.q_{95} \sim 3\right)$ and the hybrid scenario $\left(\beta_{N} \sim 2-3, q_{95} \sim 4\right)$. Both lines of research, by adopting two complementary approaches to the problem, aim at achieving a stationary scenario of the duration of $5 \mathrm{~s}$ featuring $H_{98}>0.9, W_{t h} \sim 10-12 \mathrm{MJ}$ in a domain that is accessible to JET and is relevant to ITER in terms of combined $\rho^{*}$ and $\nu^{*}$. The 
baseline activity concentrates mainly on pushing the operation towards the high current and field limits with a relaxed current profile, whereas the hybrid experiments addresses with more emphasis the advantages of operating at high $\beta_{N}$ with a shaped current profile and $q_{0}>1$. The performances of both scenarios are illustrated in figure 2 , where we plot the plasma neutron yield as a function of the stored energy. It can be seen that both baseline and hybrid plasma approach a maximum yield of $\sim 3 \cdot 10^{16}$ neutrons/s, albeit for different values of the stored energy, indicating that, while the baseline scenario has achieved higher absolute confinement, the hybrid scenario is more efficient in converting stored energy into fusion power. A further difference between baseline and hybrid scenario is the origin of the neutrons produced. In the baseline scenario, because of the higher plasma density and the shallower penetration of the neutral beams, the fraction of thermonuclear neutrons is $\sim 45 \%$ of the total yield, whereas, in the hybrid scenario, since the plasma density is lower and the neutral beams can penetrate better into the plasma core, the thermonuclear reactions account for $\sim 35 \%$ of the total yield. The non-thermal contribution, due to the fast particle population generated by NBI and ICRH, is enhanced, in both baseline and hybrid scenarios, by $\sim 7 \%$ because of the synergy between beams and ICRH [11, 12, 13, 14].

Detail of the best performing baseline plasma are shown in figure 3 , where we show the NBI, and ICRH heating power, the bulk plasma radiated power, the core ion and electron temperature, the core and edge line averaged plasma electron density, the BeII emission (indicative of the plasma ELM activity), the plasma diamagnetic energy content, the normalised $\beta$, the gas fuelling rate and the total neutron yield. In this discharge, at $3 \mathrm{MA} / 2.8 \mathrm{~T}$ with injected power of $\sim 28 \mathrm{MW}$ of $\mathrm{NBI}$ and $\sim 5 \mathrm{MW}$ of ICRH, $H_{98} \sim 1.05$ (corrected for the contribution of the ICRH fast ion pressure) and a neutron yield of $\sim 2.7 \cdot 10^{16}$ neutrons/s were obtained for $>5$ energy confinement times $(\sim 1.5$ s). These results are achieved by lowering the edge neutral particle throughput at high power, thus accessing low collisionality at the H-mode pedestal, high core confinement and high global performance. Indeed, the lowest particle throughput was achieved by means of a combination of gas and ELM pacing D pellets injection, which prevented heavy impurity accumulation with a more modest deterioration of the confinement than with gas fuelling alone. The ELM pacing pellets cause the nature of the ELMs to change radically from regular type-I ELM with a well-defined frequency to more erratic type-I ELMs with a compound character and without a clearly defined frequency. This is true also for bigger fuelling pellets reaching further into the plasma and with a visible effect on the fuelling and the density profile, used early in the experimental campaign to test their fuelling and ELM pacing capabilities. The physics underlying this achievement is still under investigation, with more experiments planned in the coming campaign addressing the effect of pacing pellets injection on the ELM behaviour, on the flushing of the impurities and, more in general, on the plasma performance. A transport analysis based on a database of recent baseline pulses suggests that, at lower collisionality and higher NBI power, a synergy may exist between higher $T_{i} / T_{e}$, ITG stabilization and central NBI ion heating, which could explain the improved performance [15]. In addition, 
high performing baseline plasma exhibit higher edge and core rotation than the worse performing ones. However, a clear causal relation between these effects has not been established yet and it is not clear how changes affecting the plasma scrape-off layer (SOL) or the region close to the separatrix can propagate and result in a better core confinement. The baseline experiment confirmed also that high ICRH power and an optimised fuelling scheme to obtain good power coupling to the plasma are essential to control the accumulation of $\mathrm{W}$ in the plasma core.

However interesting, the record baseline plasma deviates somehow from the typical baseline route to high confinement insofar it has a higher than average $\beta_{N}$ (2.2 rather than 1.8) and shows signs of MHD activity (NTMs) after $1.5 \mathrm{~s}$ into the high-performance phase. Moreover, it is not clear whether it can be extrapolated to higher current (4 MA or above) due to the limited amount of heating power available on JET (which will limit the value of $\beta_{N}$ achievable at higher current and field) and the fact that at higher current the plasma density is also higher, $T_{i} / T_{e}$ will be $\sim 1$ and the beneficial effect of operating in a regime with $T_{i}>T_{e}$ could be lost. Therefore, other discharges at $3 \mathrm{MA}$ but lower $\beta_{N}(\sim 1.8)$ are being considered as potential candidates for a baseline D-T scenario at high current. One such discharge is shown in figure 4 and, although producing only $1.5 \cdot 10^{16}$ neutrons/s it has a potential to be extrapolated, at constant $\beta_{N}$, to $4 \mathrm{MA} /$ $3.7 \mathrm{~T}$ and $40 \mathrm{MW}$ of additional heating power and give $\sim 5 \cdot 10^{16}$ neutrons/s.

As mentioned previously, similar results in terms of neutron yield were obtained at reduced plasma current in the hybrid scenario (2.2-2.5 MA/2.8-2.9 T). Details of one of the best performing hybrid plasmas are shown in figure 5 . In this discharge, at $2.2 \mathrm{MA} / 2.8 \mathrm{~T}$ with injected power of $27 \mathrm{MW}$ of NBI and $5 \mathrm{MW}$ of ICRH, $H_{98} \sim 1.15$ (corrected for the contribution of the ICRH fast ion pressure), $\beta_{N} \sim 2.5$ and a neutron yield of $2.6 \cdot 10^{16}$ neutrons/s for $\sim 1 \mathrm{~s}$ were obtained. It is interesting to note that initial attempts at producing high-performance plasmas in hybrid scenario over an extended time window were hampered by the appearance of hot spots on the the divertor tiles. This problem was solved by careful optimization of the strike point position. However, even after optimizing the divertor configuration, some of the best performing plasmas were limited in duration by the appearance of hot spots on the Be tiles in the main chamber.

The results of the baseline and the hybrid scenarios have been the object of an extensive activity of code validation and modelling [16]. In particular, semi-empirical transport models such as the Bohm/gyro-Bohm transport model [17] and physics-based transport models such as TGLF [18] and QuaLiKiZ [19] have been used to model existing discharges and to extrapolate their potential performance in D-T. The Bohm/gyroBohm transport model was used to perform coupled core-pedestal simulations, where consistency between core confinement and pedestal stability was achieved by iterating between core transport simulation and edge pedestal stability analysis as described in [20]. These simulations successfully reproduced experimental fusion performances and key trends of the plasma behaviour. Physics-based transport models were used to repeat some of the runs and investigate specific physics aspects affecting the transport such 
as rotation and $E \times B$ shear or fast particle turbulence stabilization. In the case of physics-based simulations the conditions at the top of the pedestal were taken from the semi-empirical modelling and no core-pedestal self-consistency was enforced.

The main results are summarized in figure 6 , where we show the expected D-T fusion power according to different models for the baseline and the hybrid scenario. The predictions were obtained assuming a 50-50 D-T mixture and the same power for $\mathrm{D}$ and $\mathrm{T}$ beams. No isotope effect on the confinement was included in the Bohm/gyroBohm modelling whereas both TGLF and QuaLiKiZ include an impact of the ion mass on the transport. All predictions fall broadly in the range 10-15 MW but there are uncertainties, quantified by the error bars in the plot, due, for example, to isotope effects, maximum current achievable in each scenario and model adopted for the calculation of the bootstrap current. These uncertainties will be investigated in the next experimental campaigns, where the database of $\mathrm{D}$ plasmas will be extended to higher current, magnetic field and heating power, thus providing a wider basis for model validation in D. Moreover, D plasmas will be replicated in $\mathrm{T}$ to investigate the physics of isotope effects that can affect the SOL, edge and core properties and shed some light on the performance that can be expected in $\mathrm{D}-\mathrm{T}$.

The extension of the duration of the stationary, high-performance phase to 5 s poses challenges common to both scenarios and scenario specific. The first problem, common to baseline and hybrid scenario, is the control of the divertor power load. This was systematically addressed in the hybrid scenario, initially by sweeping the strike point on the divertor tile. By optimizing the strike point central position on the divertor tile and the sweeping amplitude, it was demonstrated that high power operations $\left(P_{I N}=30\right.$ MW for $5 \mathrm{~s}$ ) are compatible with a sweeping amplitude of $3.5 \mathrm{~cm}$. In future experiments, sweeping up to $7 \mathrm{~cm}$ is envisaged and modelling suggests that this may be sufficient for $40 \mathrm{MW}$ for $5 \mathrm{~s}$ [21]. Moreover, in the hybrid scenario experiments, Ne seeding was also used as an additional method to mitigate the divertor heat load and avoid exceeding the divertor tile temperature limit by increasing the fraction of power radiated at the plasma edge and reducing the conductive power load. Although efficient in reducing the temperature of the divertor tile by a factor of three, Ne had the detrimental effect of increasing the central density thus reducing the central temperature and resulting in a non-negligible penalty on the fusion yield, which would be weaker in high temperature plasmas if thermal reactions are dominant. This is shown in figure 7 where we plot the temperature rise of the divertor tile, the plasma $\beta_{N}$ and the neutron rate as a function of the Ne seeding rate. It can be seen that, while the confinement remains constant, the neutron yield drops by nearly $40 \%$ when the Ne seeding is increased to $5 \cdot 10^{21} 1 / \mathrm{s}$ to limit the temperature increase of the divertor tile to $200{ }^{\circ} \mathrm{C}$. In addition, in hybrid plasmas, Ne seeding resulted in an enhanced electron density peaking, thus favouring the accumulation of heavy impurities in the core [22]. Therefore, strike point sweeping is at present the main method to handle high exhaust power, but the use of low Ne seeding is not ruled out if needed in D plasmas with higher additional power and might be reconsidered in $\mathrm{T}$ plasmas if tungsten sputtering by $\mathrm{T}$ becomes intolerable over $5 \mathrm{~s}$. 
Similarly, the baseline scenario experiments indicated that sweeping the divertor strike point by a few centimetres allowed to handle $35 \mathrm{MW}$ of additional power for $5 \mathrm{~s}$ without compromising the divertor plates. An optimization of the location and the amplitude of the sweeping could not be completed in the baseline scenario and further experiments are planned to confirm this result and to assess whether sweeping alone will be enough to handle the divertor power load or whether Ne injection will be necessary once more auxiliary power is injected in the plasma.

A second challenge posed by the extension of the stationary high-performance phase to $5 \mathrm{~s}$ is the avoidance of heavy impurity accumulation both during the plasma flat-top and the plasma termination. To address this problem, ICRH was used in combination with real time control of the ELM frequency by means of gas puff to help flush the tungsten [23]. The results are similar for both scenarios and show that ICRH located within $15 \mathrm{~cm}$ from the plasma magnetic axis reduces impurity accumulation. The impurity behaviour does not appear to be very sensitive to the minority $\mathrm{H}$ concentration [24]. Indeed, it has proved challenging to operate at low gas throughput and low ELM frequency (required to maintain high performance and avoid confinement degradation) and, at the same time, detect impurity accumulation during the flat-top early enough to be able to react and take remedial actions to prevent the discharge from disrupting. In addition, in the baseline scenario, it was observed that the $\mathrm{H}-\mathrm{L}$ transition and the plasma termination were affected by impurity accumulation because the ELM activity stops and the heavy impurities are not flushed anymore. To overcome these difficulties, several ideas will be tested in the following campaigns, notably better impurity accumulation detection and disruption prediction algorithms, real time control of the plasma $\beta$, optimisation of ICRH during the H-mode exit and the extension of ELM pacing pellet injection during the H-L transition to promote the flushing of the impurities. In the hybrid scenario the problem of impurity accumulation can be further enhanced by the tendency of the density profile to peak because of a more central beam particle source and therefore to induce impurity accumulation due to neoclassical transport [25] and a radiative collapse of the plasma. In this case, the density peaking was partially reduced by ICRH heating driving turbulence near the plasma core and flattening the density profile [26].

Another factor, specific to the hybrid scenario, that can limit the duration of the high-performance phase is the evolution of the $q$ profile towards a shape that allows the onset of MHD instabilities driven by the high $\beta_{N}$. It should be noted that, beside degrading the confinement, tearing modes in the hybrid scenario also lead to increased impurity accumulation [27]. At $\beta_{N}=2.4$ (feed-back controlled using NBI power) $\mathrm{m}=1$ MHD activity and tearing modes were avoided for $3.5 \mathrm{~s}$ using $q$ profile tailoring by means of beam timing and current overshoot. Further $q$ profile optimization for MHD stability is planned for the upcoming experimental campaigns to delay the onset of MHD activity even more and achieve a $5 \mathrm{~s}$ window of high-performance, MHD free hybrid scenario. 


\section{Scenarios for alpha particle studies}

The JET D-T campaign will provide a unique opportunity to further study alpha particle effects, such as alpha particle heating and alpha particle driven MHD, with respect to past results obtained in TFTR and JET D-T plasmas. These experiments require dedicated scenarios to ensure clear alpha particle physics observations. So far, the scenario development activity has concentrated on plasmas suitable for the study of alpha particle driven TAEs in an upcoming D-T campaign of JET, with the aim of validating the codes used for ITER, thus providing better confidence in the predictions of stability, fast particles redistribution and loss to the first wall [28, 29]. In D-T plasmas this scenario needs high plasma performance for only 1-2 s to generate a significant population of alpha particles, thus relaxing the requirement of a $5 \mathrm{~s}$ high performance phase, and deliberately avoids ICRH heating in D-T to avoid creating RF driven fast particles, which could mask the effect of the fusion-generated alpha particles. To maximize the alpha particle drive one needs to maximize $q_{0}, \beta_{\alpha}$ and the alpha particle pressure gradient, and minimize the damping provided mainly by beam fast ions.

It is worth emphasizing that this scenarios differ significantly from the baseline and hybrid scenarios designed for performance optimization and explore a separate region of the JET parameter space. In particular, they extend towards the lowest plasma densities achievable compatibly with the avoidance of the beam shine-through and feature a strongly elevated $q$ profile, with $q_{0}>1.5$. Moreover, because of the low plasma density they exhibit type-III ELMs, which are consistent with the requirement to minimise intra-ELM sputtering of the $\mathrm{W}$ divertor.

Plasmas with high additional power, high $T_{i}$ (to maximize the fusion yield), relatively high $T_{e}$ and low $n_{e}$ (to increase the alpha particle slowing-down time) were produced. The selected plasma current is $2.5 \mathrm{MA}$, which has been found to constitute a good compromise between low density operation and satisfactory alpha particle confinement. The toroidal magnetic field was set at $3.4 \mathrm{~T}$, which allowed probing the TAE stability of the best performing discharges by means of well-confined energetic hydrogen ions generated by ICRH injection at $51 \mathrm{MHz}$, for which the fundamental hydrogen cyclotron layer is located close to the magnetic axis. The NBI beam energies were optimised to minimise the shine-through at the start of the heating phase and allow access to lower density plasmas, whereas the timing of the NBI switch-on was optimised to slow down the current profile relaxation and maintain an elevated $q$ profile for the duration of the experiment.

The resulting $q$ profile exhibits an extended region of low positive shear which is favourable for the triggering of an internal transport barrier (ITB). Indeed, plasmas with clear ITBs were obtained for the first time since the installation of the ILW in JET. The presence of an ITB results in a significant enhancement of the thermonuclear contribution to the neutron rate. Central ion temperatures $\sim 13 \mathrm{keV}$ with $25 \mathrm{MW}$ of NBI power were obtained, resulting in a neutron yield $\sim 1.2 \cdot 10^{16}$ neutrons/s, with a significant thermonuclear contribution (up to $\sim 40 \%$ ). Subsequently, the NBI power is 
switched-off to suppress the TAE damping mechanism and allow the destabilisation of TAEs driven by the population (the so-called afterglow scenario, originally adopted on TFTR [30]). After the NBI switch off, with a delay consistent with the beam fast ion slowing-down time, TAE, induced by ICRH fast ions in this case, were observed in the range 100-200 kHz when $P_{I C R H} \sim 1-2 \mathrm{MW}$. Linear MHD calculations and the absence of any edge TAEs on reflectometry measurements for these discharges show that these modes are core-localised. This phenomenology is illustrated in figure 8, where we show, for a typical alpha particle scenario plasma, the NBI and ICRH injected power, the total neutron yield and the spectrogram from the Mirnov coils. It can be seen that the TAEs visible when the NBI power is $\sim 10 \mathrm{MW}$ disappear after it is increased to $\sim 25 \mathrm{MW}$. Two hundred millisecond after the NBI switch-off (at the peak of the neutron yield) TAEs driven by the fast ions generated by the ICRH reappear at $\sim 150 \mathrm{kHz}$.

Interpretative integrated simulations of the best performing discharges have been performed using various hypotheses in terms of impurity content, and then extrapolated to D-T plasmas. They predict that alpha particle normalised pressure could be comparable or even slightly larger than the one measured in successful alpha-driven TAE experiments in TFTR. We plan to develop this scenario further by performing discharges at higher NBI power in an upcoming D campaign and to consolidate extrapolations to $\mathrm{D}-\mathrm{T}$ by performing similar pulses in pure $\mathrm{T}$ plasmas before applying this scenario to the next JET D-T campaign.

\section{Conclusions}

In this paper we have described the scenario development activity carried on at JET in preparation for a D-T campaign in 2020. Good progress has been made towards reaching the key performance indicators establishing the $\mathrm{D}-\mathrm{T}$ readiness of a scenario. In particular, the baseline and hybrid scenario have demonstrated the capability of producing $\sim 2.2 \cdot 10^{16} \mathrm{D}$-D neutrons/s averaged over $5 \mathrm{~s}$, a result which, although more than a factor of two below the target of $5 \cdot 10^{16}$ neutrons/s, is consistent with the limited NBI power that was available and will be substantially improved when $32 \mathrm{MW}$ of NBI at $120 \mathrm{keV}$ and 6-8 MW of ICRH power will be reliably available. Moreover, the scenario developed for the study of alpha particle effects, has demonstrated the potential of creating a plasma with alpha particle pressure high enough to destabilize TAEs in the afterglow scenario, with a slowing-down time delay after the NBI switching-off.

In next experimental campaigns, the scenario development effort for DTE2 will continue. All three scenarios established so far will be improved with the aim of consolidating and improve existing results. In particular, once the disruptivity has been reduced to acceptable levels, the baseline scenario will be pushed towards higher current and field. The hybrid scenario parameter space will also be extended to higher field and explore the optimization of $q_{95}$ and the plasma shape while further tailoring the current density profile before the high-performance phase. A strong emphasis will be placed on improving the MHD stability of the discharge to extend the MHD free 
phase to $5 \mathrm{~s}$. Further optimization will also be the objective of the scenario dedicated to alpha particle studies. All scenarios will be replicated in $\mathrm{T}$ to investigate the impact of possible isotope effects.

Finally, we will also develop and test real time control schemes to control various plasma physics and machine operational parameters such as $\beta_{N}$, ELM frequency, plasma isotope composition and divertor temperature. A 'dud' detection system will also be developed to stop poorly performing plasma and save $\mathrm{T}$ consumption and minimize the neutron production.

In conclusion, the encouraging results obtained so far indicate that it should be possible to have successful D-T campaign on JET in 2020 in presence of an ITER-like wall and taking advantage of the widely extended set of diagnostics with respect to what was available in 1997.

\section{Acknowledgments}

This work has been carried out within the framework of the EUROfusion Consortium and has received funding from the Euratom research and training programme 20142018 and 2019-2020 under grant agreement No 633053 and from the RCUK Energy Programme (grant number EP/P012450/1). To obtain further information on the data and models underlying this paper please contact PublicationsManager@ukaea.uk. The views and opinions expressed herein do not necessarily reflect those of the European Commission.

\section{References}

[1] Joffrin E. et al Overview of the JET preparation for Deuterium-Tritium operation, in FEC 2018, Proceedings of the 27th IAEA Fusion Energy Conference (Gandhinagar, India, 22-27 October 2018) (2018) OV/1-3

[2] Keilhacker M. et al Nucl. Fusion 39 (1999) 209

[3] Jacquinot J. et al Nucl. Fusion 39 (1999) 235

[4] Figueiredo J. et al Rev. Sci. Instrum. 87 (2016) 11D443

[5] Figueiredo J. et al Rev. Sci. Instrum. 89 (2018) $10 \mathrm{~K} 119$

[6] Pasqualotto R. et al Rev. Sci. Instrum. 75 (2004) 3891

[7] Gatu-Johnson M. et al Rev. Sci. Instrum. 77 (2006) 10E702

[8] JET Team (prepared by C. Gormezano) Nucl. Fusion 39 (1999) 1875

[9] Maggi C. F. et al Plasma Phys. Control. Fusion 60 (2018) 014045

[10] Weisen H. et al Isotope dependence of confinement in JET deuterium and hydrogen plasmas, in FEC 2018, Proceedings of the 27th IAEA Fusion Energy Conference (Gandhinagar, India, 22-27 October 2018) (2018) EX/P1-4

[11] Mantsinen M. J. et al European Physical Journal Web of Conferences 157 (2017) 3032

[12] Mantsinen M. J. et al Europhysics Conference Abstracts 41F (2017) O3.110

[13] Gallart D. et al European Physical Journal Web of Conferences 157 (2017) 3015

[14] Gallart D. et al Modelling of JET hybrid plasmas with emphasis on performance of combined ICRF and NBI heating, accepted for publication in Nucl. Fusion

[15] Kim H.-T. et al Nucl. Fusion 58 (2018) 036020 


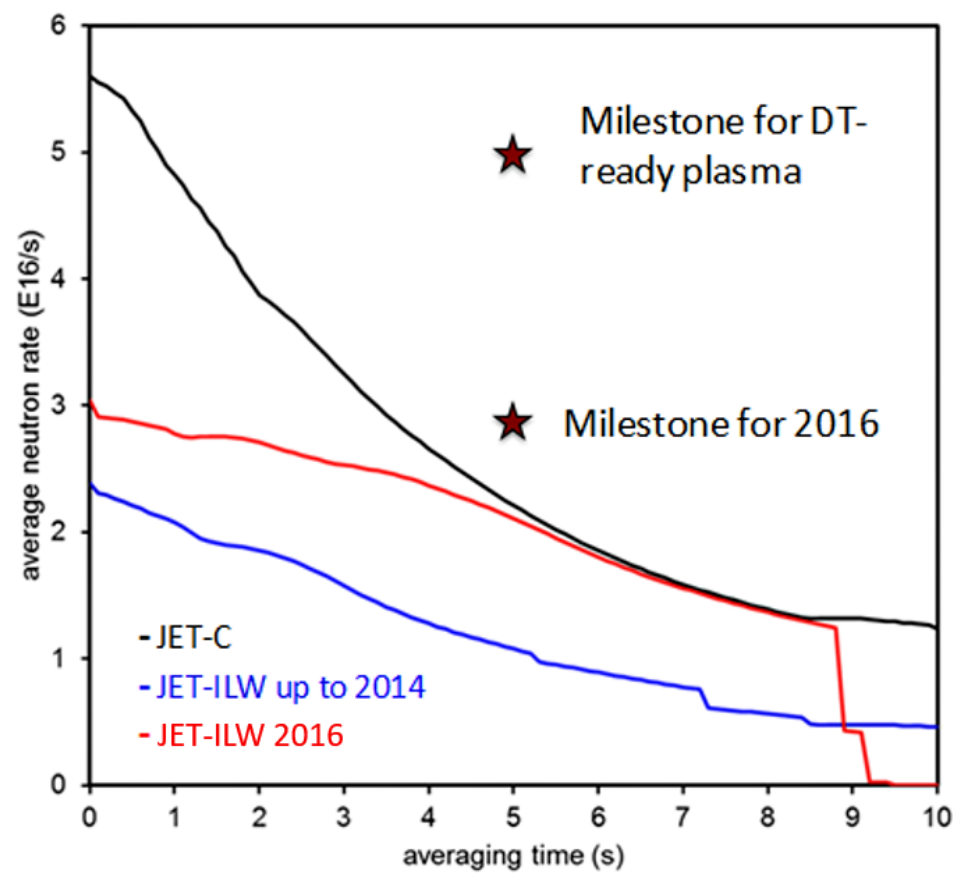

Figure 1. Average D-D neutron rate as function of the averaging time for JET's best performing shots. The stars show the milestones for 2016 and a for a D-T ready plasma.

[16] Garcia J. et al First principles and integrated modelling achievements towards trustful fusion power predictions for JET and ITER, in FEC 2018, Proceedings of the 27th IAEA Fusion Energy Conference (Gandhinagar, India, 22-27 October 2018) (2018) TH/3-1

[17] Erba M. et al Plasma Phys. Control. Fusion 39 (1997) 261

[18] Staebler G. M. et al Phys. Plasmas 12 (2005) 102508

[19] Bourdelle C. et al Plasma Phys. Control. Fusion 58 (2015) 014036

[20] Saarelma S. et al Plasma Phys. Control. Fusion 60 (2018) 014042

[21] Silburn S. et al Physica Scripta T170 (2017) 014040

[22] Frigione D. et al Impact of neon injection on electron density peaking in JET hybrid plasmas, in FEC 2018, Proceedings of the 27th IAEA Fusion Energy Conference (Gandhinagar, India, 22-27 October 2018) (2018) EX/P1-3

[23] da la Luna E. et al Impact of ELM control in JET experiments on H-Mode terminations with/without current ramp-down and implications for ITER, in FEC 2018, Proceedings of the 27th IAEA Fusion Energy Conference (Gandhinagar, India, 22-27 October 2018) (2018) EX/2-1

[24] Lerche E. et al Nucl. Fusion 56 (2016) 036022

[25] Angioni C. et al Nucl. Fusion 54 (2014) 083028

[26] Casson F. et al Predictive multi-channel flux-driven modelling to optimise ICRH tungsten control in JET, in FEC 2018, Proceedings of the 27th IAEA Fusion Energy Conference (Gandhinagar, India, 22-27 October 2018) (2018) TH/3-2

[27] Hender T. C. et al Nucl. Fusion 56 (2016) 066002

[28] Dumont R. J. et al Nucl. Fusion 58 (2018) 082005

[29] Sharapov S. et al, Plasma and diagnostics preparation for alpha-particle studies in JET DT, in FEC 2018, Proceedings of the 27th IAEA Fusion Energy Conference (Gandhinagar, India, 22-27 October 2018) (2018) EX/P1-28

[30] Nazikian R. et al Phys. Rev. Lett. 78 (1997) 2976 


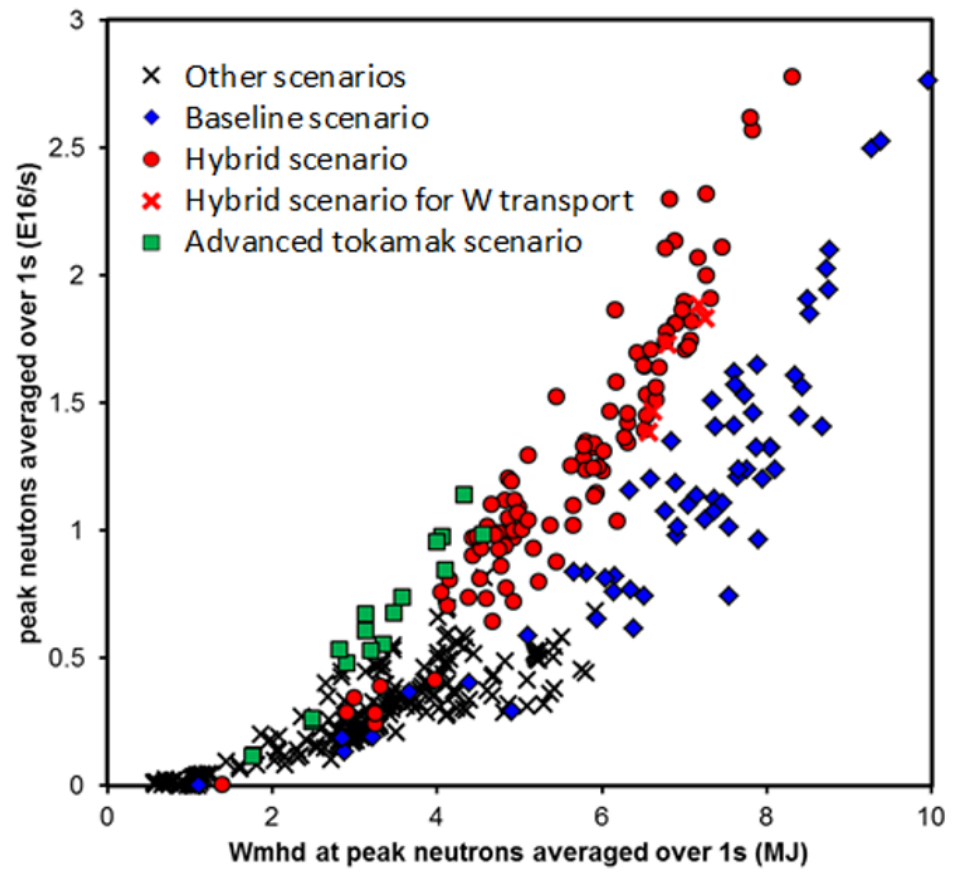

Figure 2. Neutron rate as function of plasma stored energy achieved at JET in 2016 campaigns in baseline and hybrid plasmas and in plasmas optimized for alpha particle studies. 


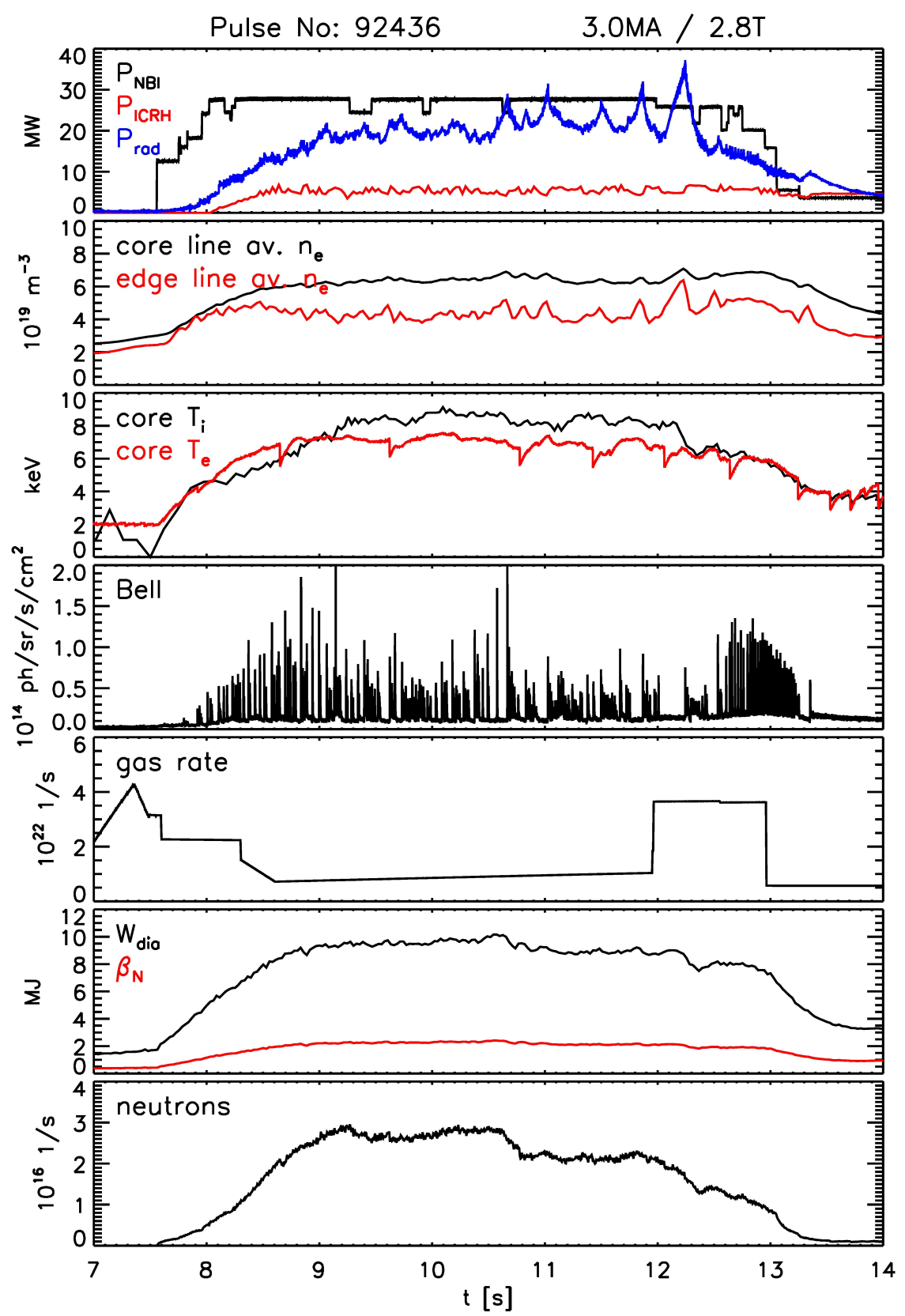

Figure 3. Time traces for the best performing JET baseline plasma. ELM pacing pellets (mass $2.1 \cdot 10^{20} \mathrm{D}$ atoms and frequency $41 \mathrm{~Hz}$ ) are injected between $7.2 \mathrm{~s}$ and $12 \mathrm{~s}$. 


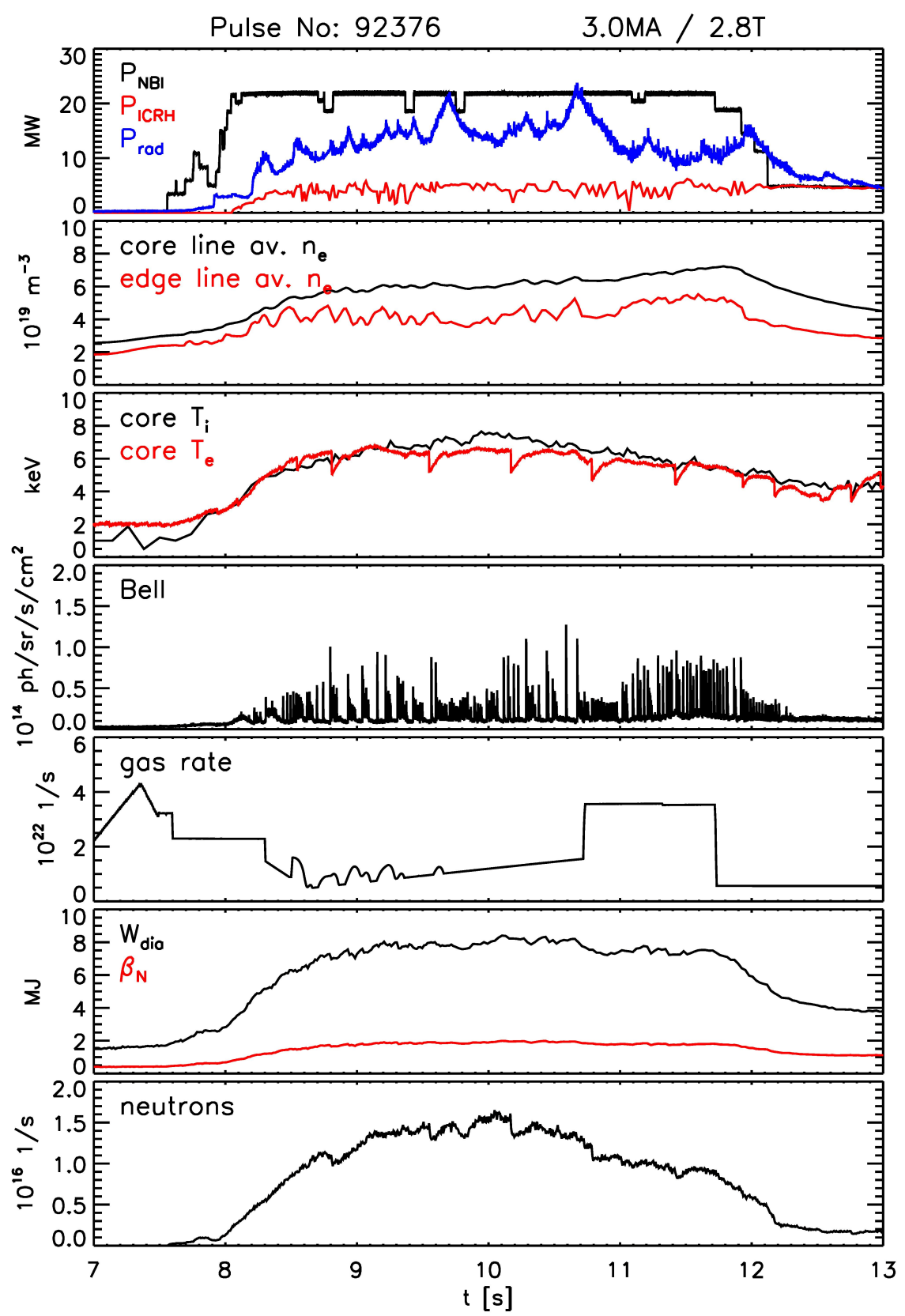

Figure 4. Time traces for an average $\beta_{N}$ JET baseline plasma, but with the potential of extrapolating to $4 \mathrm{MA}$. ELM pacing pellets (mass $2.1 \cdot 10^{20} \mathrm{D}$ atoms and frequency $41 \mathrm{~Hz}$ ) are injected between $7.2 \mathrm{~s}$ and $11.3 \mathrm{~s}$. 


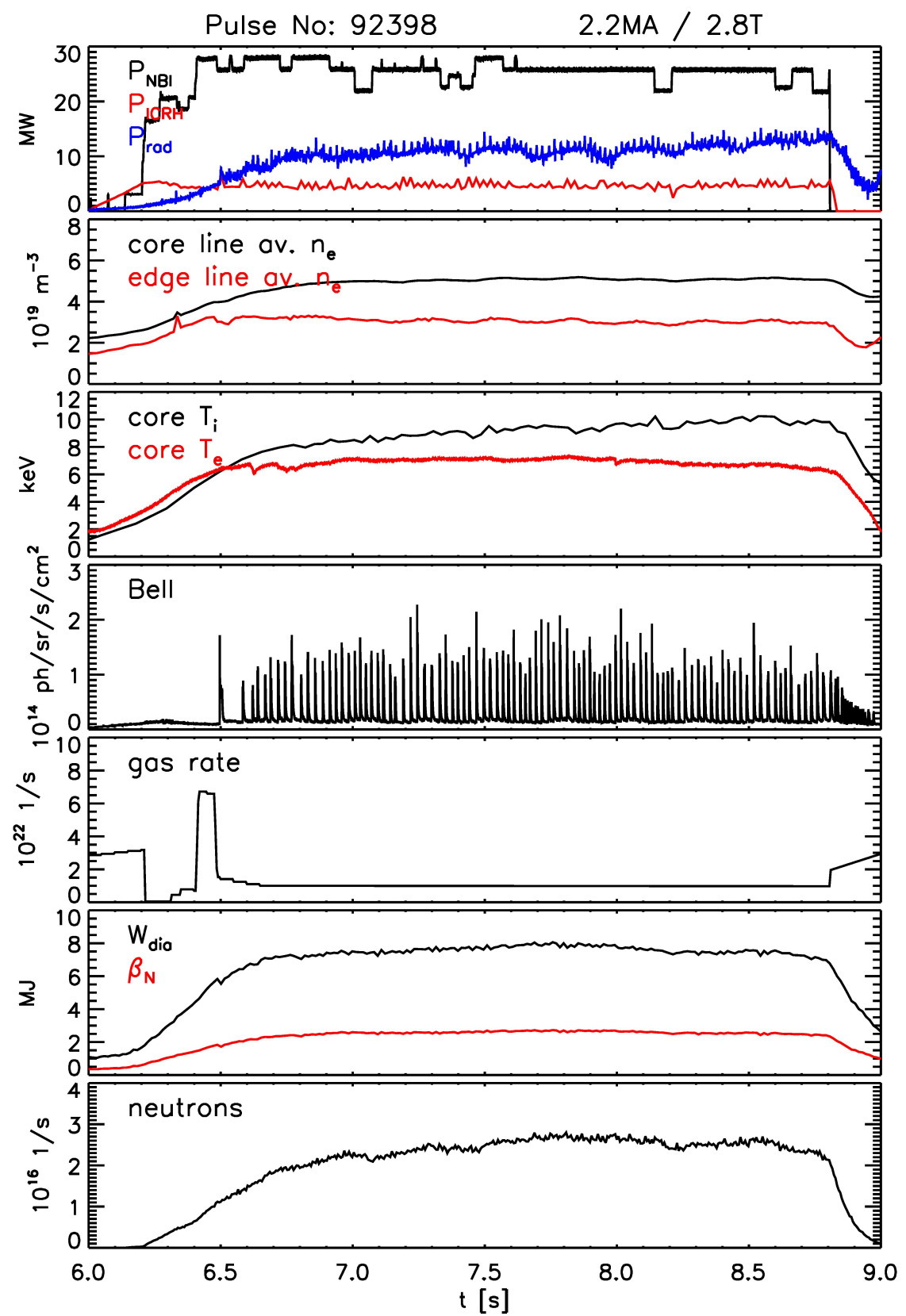

Figure 5. Time traces for the best performing JET hybrid plasma. 


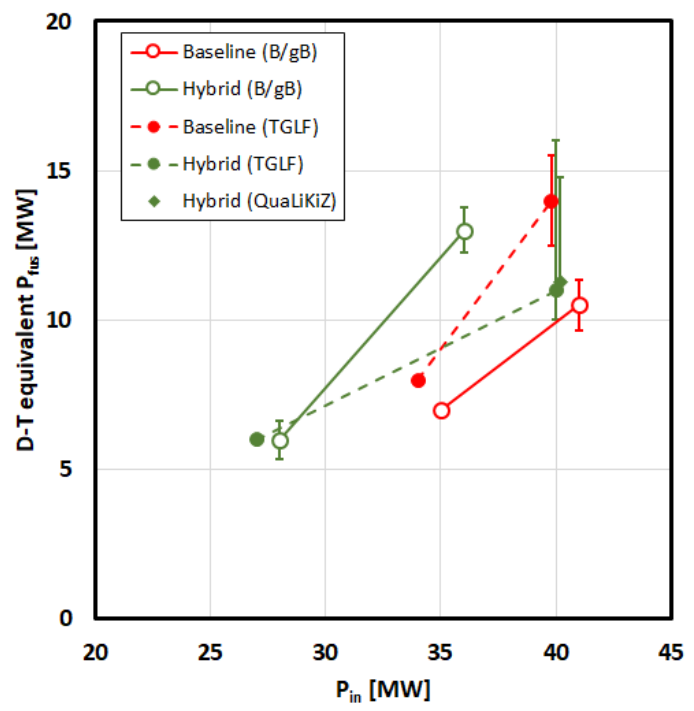

Figure 6. D-T fusion power extrapolated to higher current and additional heating power from the best baseline and hybrid plasmas. The error bars in the Bohm/gyroBohm simulations quantify the uncertainties on the maximum achievable current (for the baseline case) and the estimate of the bootstrap current (for the hybrid case). The error bars in the QuaLiKiZ and TGLF simulations quantify the uncertainty on the isotope effect on the confinement.

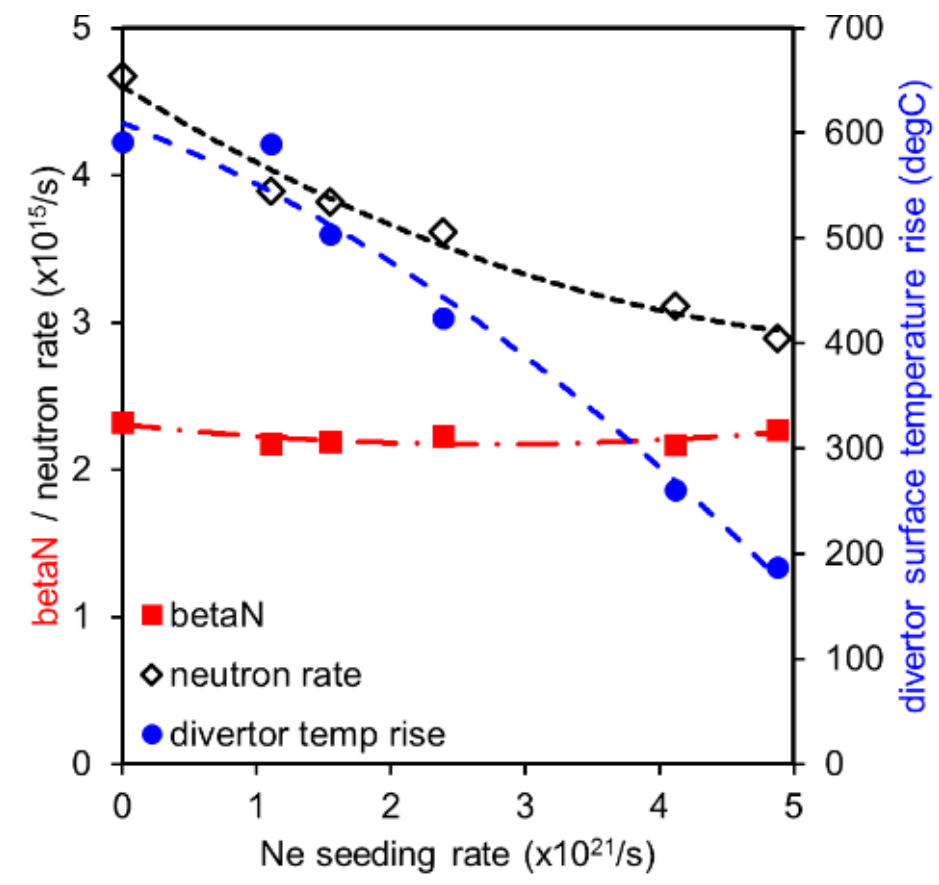

Figure 7. $\beta_{N}$, neutron rate and divertor temperature rise for a series of hybrid plasmas with increasing Ne seeding rate. 


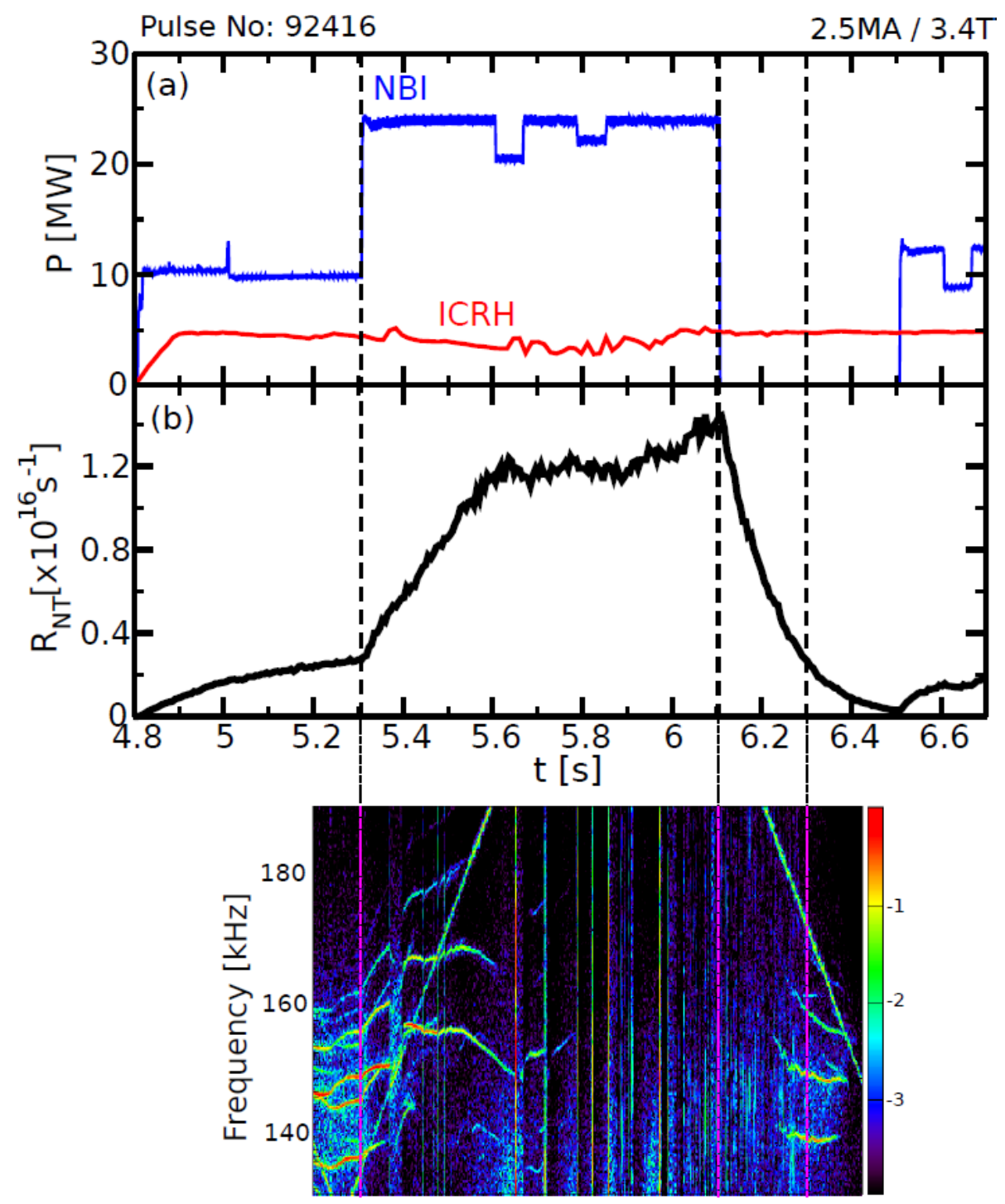

Figure 8. Time traces of NBI and ICRH power, D-D neutron rate and Mirnov coils spectrogram for a JET plasma developed for the observation of alpha particle driven TAEs. ICRH induced TAEs appear with a slowing-down time delay after the switching-off of the beams. 